\title{
A Pressure Transient Model for Power-Law Fluids in Porous Media Embedded with a Tree-Shaped Fractal Network
}

\author{
Xiao-Hua Tan, ${ }^{1}$ Jian-Yi Liu, ${ }^{1}$ Jia-Hui Zhao, ${ }^{1}$ Xiao-Ping Li, ${ }^{1}$ \\ Guang-Dong Zhang, ${ }^{1}$ and Chuan Tang ${ }^{2}$ \\ ${ }^{1}$ State Key Laboratory of Oil and Gas Reservoir Geology and Exploitation, Southwest Petroleum University, Xindu Road 8, \\ Chengdu 610500, China \\ ${ }^{2}$ Shell China Exploration \& Production Co. Ltd., 8F Yanlord Landmark Office Building, No. 1, Section 2, Renmin South Road, \\ Chengdu 610500, China
}

Correspondence should be addressed to Xiao-Hua Tan; xiaohua-tan@163.com

Received 4 November 2013; Accepted 14 January 2014; Published 24 February 2014

Academic Editor: Hai Yu

Copyright (C) 2014 Xiao-Hua Tan et al. This is an open access article distributed under the Creative Commons Attribution License, which permits unrestricted use, distribution, and reproduction in any medium, provided the original work is properly cited.

\begin{abstract}
This work studies the pressure transient of power-law fluids in porous media embedded with a tree-shaped fractal network. A pressure transient model was created based on the fractal properties of tree-shaped capillaries, generalized Darcy's law and constitutive equation for power-law fluids. The dimensionless pressure model was developed using the Laplace transform and Stehfest numerical inversion method. According to the model's solution, the bi-logarithmic type curves of power-law fluids in porous media embedded with a tree-shaped fractal network are illustrated. The influences of different fractal factors and Powerlaw fluids parameters on pressure transient responses are discussed.
\end{abstract}

\section{Introduction}

Power-law fluids flow in porous media has always been a subject of great interest owing to its fundamental and pragmatic significance. Consequently, over the years, a voluminous amount of research has been conducted to gain insight into phenomena that is related to fluids flow. Traditionally, numerical methods have played a significant role in the analysis of power-law fluids flow in porous media. Lopez et al. [1] determined the flow of power-law fluids in porous media using network models. Through the use of numerical methods, a network model is capable of predicting each of the parameters associated with a pore, such as pressure drop, velocity, and superficial viscosity. Network model predictions are superior to those that are based on simplified capillary bundle models. Kumari and Nath [2] used numerical methods to study non-Darcy mixed convection of power-law fluids in porous media. Orgéas et al. [3] created a flow model for power-law fluids in anisotropic porous media at the low-pore Reynolds number. Pascal [4] researched the transient flow of power-law fluids in a porous incline using the Kármán momentum integral method. Non-Newtonian constitutive equations, used as one of the numerical methods, have been incorporated into the conventional lattice Boltzmann method (LBM) to simulate flow of power-law fluids [59]. However, the LBM demands tedious calculations and necessitates accuracy of model. These constraints limit the LBM's ability to describe flow of power-law fluids in porous media.

Yun et al. [10] have proposed the starting pressure gradient model for the flow of Bingham fluids in fractal porous media, based on the fractal nature of pore size distribution in porous media. They also analyzed the effect of capillary pressure on the starting pressure gradient. Results showed that the effect of capillary pressure on the starting pressure gradient at a low porosity is significant and cannot be neglected. However, at high porosity, the effect of capillary pressure on the starting pressure gradient is found to be negligible. $\mathrm{Li}$ and $\mathrm{Yu}$ [11] proposed a starting pressure gradient model for Bingham fluids flow in fractal networks, which could be used to analyze the influence of network microstructural parameters on the starting pressure gradient and on network permeability. The model can also be used to study Bingham fluids flow in dual porosity media. The theory proposed by 


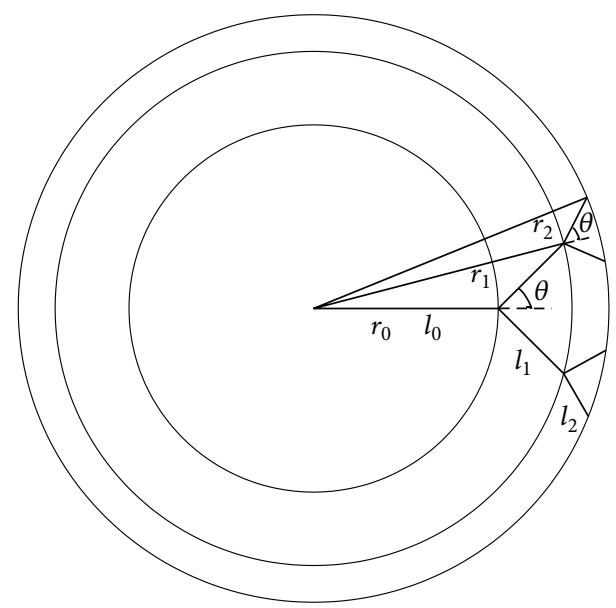

FIgURE 1: Porous media embedded with a tree-shaped fractal network

Yun et al. [10] and Li and Yu [11] was further developed by Wang and Yu [12]. They derived the starting pressure gradient model for Bingham fluids flow in tree-shaped fractal porous media by embedding the tree-shaped fractal network into porous media. Based on the tree-shaped fractal model, Wang et al. [13] proposed a starting pressure gradient model for Bingham fluids flow in porous media that is embedded with randomly distributed tree-shaped fractal networks.

\section{Physical Modeling}

Figure 1 shows a coordinate system for radial flow toward the center point, $O$, from the external boundary. The center point, $O$, has an internal boundary, $r_{\text {in }} . h$ is the system thickness. The coordinate system consists of two parts: the tree-shaped fractal network and the porous media. During a tree-shaped fractal network generation, the branches on each level must end up on the same circle with all circles having the same center of origin, O. $N$ tubes, which start at $O$, make up the tree-shaped fractal network. The tube's initial length and diameter are $l_{0}$ and $d_{0}$, respectively. The double branches $(b=$ $2)$, whose angles are $\theta(\theta<\pi / 2)$ and total network branch levels are $M$, are applied in this network. Furthermore, two scale factors are used in this fractal network, length ratio, $\alpha$, and diameter ratio, $\beta$.

The physic model assumptions are as follows.

(1) The branch tube in tree-shaped fractal network is assumed to be smooth and tube wall thickness is ignored.

(2) The porous media is divided into $M$ annular sections by a tree-shaped fractal network.

(3) Each of the tree-shaped fractal network section's properties is different, but each of the porous media section's properties is identical.

(4) Porous media permeability is much lower than treeshaped fractal network permeability, and fluids only flow to the center point, $O$, through the tree-shaped fractal network.
(5) Rock and liquid are considered slightly compressible, with each having a constant and small compressibility.

(6) Isothermal and single direction flow is considered.

(7) Capillary pressure and gravity effects are neglected.

(8) Non-Newtonian fluids obey the power-law principle and are considered as pseudoplastic fluids $(0<n<$ $1)$.

(9) The internal boundary is considered to have constant flow rate, while the external boundary is considered to be closed or at constant pressure.

(10) At time $t=0$, pressure is uniformly distributed in all system and is equal to initial pressure $\left(p_{i}\right)$.

\section{Mathematical Modeling}

3.1. Flow Parameters in the Tree-Shaped Fractal Network. The flow velocity through a single tube for power-law fluids is given by [14]

$$
v=\frac{d^{1+(1 / n)}}{2^{1+(2 / n)} m^{1 / n}(3+(1 / n))}\left(-\frac{d p}{d L}\right)^{1 / n},
$$

where $\tau$ is shear stress, $m$ is the consistency index, $\dot{\gamma}$ is shear rate, and $n$ is the power index.

Ikoku and Ramey Jr [15] defined the flow velocity for power-law fluids as

$$
v=\left(-\frac{K}{\mu_{\mathrm{eff}}} \frac{d p}{d L}\right)^{1 / n},
$$

where $K$ is permeability and $\mu_{\text {eff }}$ is effective viscosity.

Substituting (1) into (2), permeability for power-law fluids in a single tube can be expressed by effective viscosity:

$$
K=\frac{\mu_{\mathrm{eff}} d^{n+1}}{2^{n+2} m(3+(1 / n))^{n}} .
$$

When $n=1$ and $m=\mu_{\text {eff }}=\mu$ in (3), the permeability expression for power-law fluids reduces to that for Newtonian fluids

$$
K=\frac{d^{2}}{32}
$$

which agrees with the research result of Xu et al. [16].

To characterize the branching structures, let the length and diameter of a typical branch at some intermediate level $k(k=0,1, \ldots, M)$ be $d_{k}$ and $l_{k}$, respectively. Two scale factors length ratio, $\alpha$, and diameter ratio, $\beta$, are defined as

$$
\begin{aligned}
& \alpha=\frac{l_{k+1}}{l_{k}}, \\
& \beta=\frac{d_{k+1}}{d_{k}} .
\end{aligned}
$$

For the $k$ th level tube, length and diameter are given by

$$
\begin{gathered}
l_{k}=\alpha^{k} l_{0}, \\
d_{k}=\beta^{k} d_{0} .
\end{gathered}
$$


The interface radius is defined as the distance from the internal boundary to each section's boundary [16]:

$$
r_{k}=l_{0}\left[1+\frac{\alpha\left(1-\alpha^{k}\right) \cos \theta}{1-\alpha}\right] .
$$

The external flow radius is defined as the distance from the internal boundary to the external boundary:

$$
r_{\mathrm{ex}}=r_{M}=l_{0}\left[1+\frac{\alpha\left(1-\alpha^{M}\right) \cos \theta}{1-\alpha}\right] \text {. }
$$
by

The single tube permeability in $k$ th section is expressed

$$
K_{f s k}=\frac{\mu_{\mathrm{eff}} d_{k}^{n+1}}{2^{n+2} m(3+(1 / n))^{n}} .
$$

Considering $k$ th section in tree-shaped fractal network tortuosity $T_{k}$, the single tube permeability in $k$ th section can be expressed as

$$
K_{f s k}=\frac{\mu_{\mathrm{eff}} d_{k}^{n+1}}{2^{n+2} m T_{k}(3+(1 / n))^{n}}
$$

where

$$
T_{k}=\frac{l_{k}}{r_{k}-r_{k-1}}= \begin{cases}1 & k=0 \\ \frac{1}{\cos \theta} & k>0\end{cases}
$$

Substituting (7) into (11), the permeability in $k$ th section of tree-shaped fractal network is

$$
K_{f k}= \begin{cases}\frac{N \mu_{\mathrm{eff}} d_{k}^{n+1}}{2^{n+2} m(3+(1 / n))^{n}} & k=0 \\ \frac{N \mu_{\mathrm{eff}} b^{k} \beta^{2 k} d_{k}^{n+1} \cos \theta}{2^{n+2} m T_{k}(3+(1 / n))^{n}} & k>0 .\end{cases}
$$

The total system volume in the $k$ th section of tree-shaped fractal network can be calculated by

$$
V_{k}= \begin{cases}\pi h r_{0}^{2} & k=0 \\ \pi h\left(r_{k}^{2}-r_{k-1}^{2}\right) & k>0\end{cases}
$$

The volume in the $k$ th section of tree-shaped fractal network can be calculated by

$$
V_{f k}=\frac{N \pi b^{k} \alpha^{k} \beta^{2 k} l_{0} d_{0}^{2}}{4} .
$$

The tree-shaped fractal network porosity in the $k$ th section of tree-shaped fractal network can be obtained by dividing (15) by (14):

$$
\phi_{f k}=\frac{V_{f k}}{V_{k}}= \begin{cases}\frac{N d_{0}^{2}}{4 h l_{0}} & k=0 \\ \frac{N b^{k} \alpha^{k} \beta^{2 k} l_{0} d_{0}^{2}}{4 h\left(r_{k}^{2}-r_{k-1}^{2}\right)} & k>0 .\end{cases}
$$

Under the condition of double branches $(b=2)$, the permeability, $K_{f k}$, and porosity, $\phi_{f k}$, in the $k$ th section of treeshaped fractal network are equal; that is,

$$
\begin{aligned}
& K_{f k}=K_{f(k+1)} \\
& \phi_{f k}=\phi_{f(k+1)} .
\end{aligned}
$$

Substituting (13) and (16) into (17), we can obtain $b=2$, $\alpha=1$, and $\beta=0.707$.

3.2. Mathematical Model. According to the physical model, the mathematical pressure transient model for power-law fluids in porous media embedded with a tree-shaped fractal network can be described as follows.

Governing differential equations for tree-shaped fractal network [17]:

$$
\begin{gathered}
\frac{K_{k}}{n \mu_{\mathrm{eff}}}\left(\frac{q}{2 \pi h}\right)^{1-n} r^{n-1}\left(\frac{\partial^{2} p_{f k}}{\partial r^{2}}+\frac{n}{r} \frac{\partial p_{f k}}{\partial r}\right) \\
+\frac{a K_{m}}{\mu_{\mathrm{eff}}}\left(p_{m k}-p_{f k}\right) \\
=\left(\phi C_{t}\right)_{f k} \frac{\partial p_{f k}}{\partial t} \quad r_{k-1} \leq r \leq r_{k} .
\end{gathered}
$$

Governing differential equations for porous media [17]:

$$
-\frac{a K_{m}}{m u_{\mathrm{eff}}}\left(p_{m k}-p_{f k}\right)=\left(\phi C_{t}\right)_{m} \frac{\partial p_{m k}}{\partial t} \quad r_{k-1} \leq r \leq r_{k} .
$$

Initial condition:

$$
p_{f k}(r, 0)=p_{m k}(r, 0)=p_{i} \quad(k=0,1, \ldots, M) .
$$

Interface connecting conditions of each section, pressure continuity [18]:

$$
\left.p_{f k}\right|_{r=r_{k}}=\left.p_{f(k+1)}\right|_{r=r_{k}} \quad(k=0,1, \ldots, M-1) .
$$

Interface connecting conditions of each section, rate continuity [18]:

$$
\left.\frac{\partial p_{f k}}{\partial r}\right|_{r=r_{k}}=\left.\frac{k_{f(k+1)}}{k_{f k}} \frac{\partial p_{f(k+1)}}{\partial r}\right|_{r=r_{k}} \quad(k=0,1, \ldots, M-1) .
$$

Internal boundary condition [19]:

$$
p_{\text {in }}=\left.p_{f 0}\right|_{r=r_{\text {in }}} .
$$

External boundary condition (infinite):

$$
p_{f M}(r \longrightarrow \infty, t)=p_{i} .
$$

External boundary condition (constant pressure):

$$
p_{f M}\left(r=r_{M}, t\right)=p_{i} .
$$

External boundary condition (closed):

$$
\left.\frac{\partial p_{f M}}{\partial r}\right|_{r=r_{M}}=0
$$


3.3. Solution to Mathematical Model. The dimensionless mathematical model for power-law fluids in porous media embedded with a tree-shaped fractal network is shown in the Appendix B. Introduce the Laplace transform based on $t_{D}$. The dimensionless mathematical model in Laplace space is as follows.

Governing differential equations for tree-shaped fractal network:

$$
\begin{gathered}
\frac{d^{2} \bar{p}_{D f k}}{d r_{D}^{2}}+\frac{n}{r_{D}} \frac{d \bar{p}_{D f k}}{d r_{D}}+\lambda_{k} r_{D}^{1-n}\left(\frac{K_{f k}}{K_{m}} \bar{p}_{D m k}-\bar{p}_{D f k}\right) \\
=\omega_{k} r_{D}^{1-n} \frac{K_{f 0}}{K_{f k}} z \bar{p}_{D f k} \quad r_{D(k-1)} \leq r_{D} \leq r_{D k} .
\end{gathered}
$$

Governing differential equations for porous media:

$$
\begin{array}{r}
-\lambda_{k}\left(\bar{p}_{D m k}-\frac{K_{m}}{K_{f k}} \bar{p}_{D f k}\right)=\left(1-\omega_{k}\right) \frac{K_{f 0}}{K_{f k}} z \bar{p}_{D m k} \\
r_{D(k-1)} \leq r_{D} \leq r_{D k} .
\end{array}
$$

Initial condition:

$$
\bar{p}_{D f k}\left(r_{D}, 0\right)=\bar{p}_{D m k}\left(r_{D}, 0\right)=0 \quad(k=0,1, \ldots, M) \text {. }
$$

Interface connecting conditions of each zone, pressure continuity:

$$
\left.\bar{p}_{D f k}\right|_{r_{D}=r_{D k}}=\left.\bar{p}_{D f(k+1)}\right|_{r_{D}=r_{D k}} \quad(k=0,1, \ldots, M-1) .
$$

Interface connecting conditions of each zone, rate continuity:

$$
\begin{array}{r}
\left.\frac{d \bar{p}_{D f k}}{d r_{D e}}\right|_{r_{D}=r_{D k}}=\left.\frac{K_{f(k+1)}}{K_{f k}} \frac{d \bar{p}_{D f(k+1)}}{d r_{D}}\right|_{r_{D}=r_{D k}} \\
(k=0,1, \ldots, M-1) .
\end{array}
$$

Internal boundary condition:

$$
\left.\frac{d \bar{p}_{D_{\mathrm{in}}}}{d r_{D}}\right|_{r_{D}=1}=-1
$$

External boundary condition (infinite):

$$
\bar{p}_{D f M}\left(r_{D} \longrightarrow \infty, z\right)=0 \text {. }
$$

External boundary condition (constant pressure):

$$
\bar{p}_{D f M}\left(r_{D}=r_{D M}, z\right)=0 \text {. }
$$

External boundary condition (closed):

$$
\left.\frac{d \bar{p}_{D f M}}{d r_{D}}\right|_{r_{D}=r_{D M}}=0
$$

Substituting (28) into (29), we can obtain

$$
\begin{array}{r}
\frac{d^{2} \bar{p}_{D f k}}{d r_{D}^{2}}+\frac{n}{r_{D k}} \frac{d \bar{p}_{D f k}}{d r_{D}}-r_{D k}^{1-n} z S(z) \bar{p}_{D f k}=0 \\
(k=0,1, \ldots, M) .
\end{array}
$$

In (36), The dimensionless interface radius, $r_{D k}$, and the function, $S_{k}(z)$, can be directly expressed by the tree-shaped fractal network factors.

The dimensionless interface radius expression can be derived by substituting (8) into (A.3):

$$
r_{D k}=\frac{l_{0}}{r_{\text {in }}}\left[1+\frac{\alpha\left(1-\alpha^{k}\right) \cos \theta}{1-\alpha}\right] \text {. }
$$

The expression of the function $S_{k}(z)$ is as follows:

$$
S_{k}(z)=\frac{\left(K_{f 0} / K_{f k}\right) \lambda_{k}\left(1-\omega_{k}\right)}{\left(K_{f 0} / K_{f k}\right)\left(1-\omega_{k}\right) z+\lambda_{k}}+\frac{K_{f 0}}{K_{f k}} \omega_{k},
$$

where

$$
\frac{K_{f 0}}{K_{f k}}=\left(b \beta^{2}\right)^{-k} .
$$

The fluid capacitance coefficient, $\omega_{k}$, can be obtained by substituting (16) into (A.5), and the interporosity flow coefficient, $\lambda_{k}$, can be obtained by substituting (13) into (A.6).

The general solution of $\bar{p}_{D f k}$ in (36) is calculated

$$
\begin{array}{r}
\bar{p}_{D f k}=A_{k} f_{I}\left(r_{D k}, S_{k}(z)\right)+B_{k} f_{K}\left(r_{D k}, S_{k}(z)\right) \\
(k=0,1, \ldots, M),
\end{array}
$$

where

$$
\begin{gathered}
f_{I}\left(r_{D k}, S_{k}(z)\right)=r_{D k}^{(1-n) / 2} I_{v}\left(r_{D k}^{(3-n) / 2} \frac{2 \sqrt{S_{k}(z)}}{3-n}\right), \\
f_{K}\left(r_{D k}, S_{k}(z)\right)=r_{D k}^{(1-n) / 2} K_{v}\left(r_{D k}^{(3-n) / 2} \frac{2 \sqrt{S_{k}(z)}}{3-n}\right), \\
v=\frac{(1-n)}{(3-n)} .
\end{gathered}
$$

Then, the derivative of $\bar{p}_{D f k}$ in (40) can be expressed as

$$
\begin{array}{r}
\frac{d \bar{p}_{D f k}}{d r_{D}}=A_{k} \frac{f_{I}\left(r_{D k}, S_{k}(z)\right)}{d r_{D}}+B_{k} \frac{f_{K}\left(r_{D k}, S_{k}(z)\right)}{d r_{D}} \\
(k=0,1, \ldots, M) .
\end{array}
$$

\section{Flow Behavior Characteristics}

4.1. Flow Regime Recognition. $\bar{p}_{D f k}, A_{k}$ and $B_{k}(k=0$, $1, \ldots, M)$ can be obtained by solving the general solution equations (40) and (42) and definite condition equations (32)-(35). We can obtain dimensionless internal boundary pressure in real space, $p_{D_{i n}}$, by the Stehfest numerical inversion method. The bilogarithmic type curves for powerlaw fluids in porous media embedded with a tree-shaped fractal network can be illustrated. In a condition of closed external boundary, the transient flow process, which has four flow regimes, can be clearly shown (Figure 2). The full and dashed lines represent pressure and pressure derivative curves, respectively. 


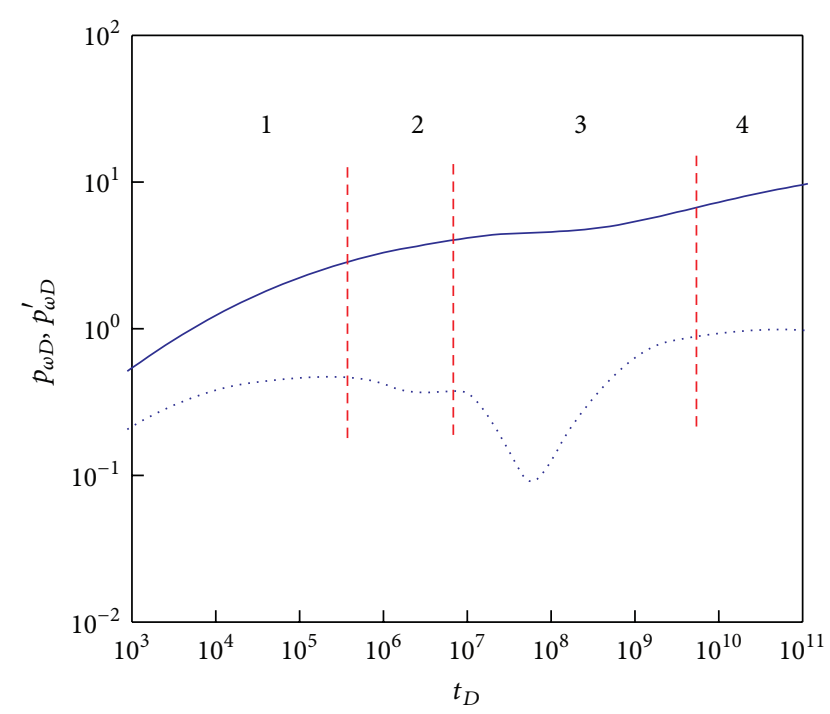

FIGURE 2: Pressure type curves for power-law fluids in porous media embedded with a tree-shaped fractal network $(n=0.9, \alpha=1, \beta=$ 0.707, $\theta=1, N=4, M=10, l_{0}=10 \mathrm{~m}, d_{0}=0.05 \mathrm{~m}, r_{\text {in }}=0.1 \mathrm{~m}$, $h=5 \mathrm{~m}, C_{m t}=2.2 \times 10^{-5} \mathrm{MPa}^{-1}$, and $\left.C_{f t}=1 \times 10^{-4} \mathrm{MPa}^{-1}\right)$.

Regime 1 is interporosity flow in tree-shaped fractal network regime. Power-law fluids start to flow in tree-shaped fractal network. Dimensionless pressure increases rapidly before slowing slightly, because it is hard to flow for power-law fluids $(0<n<1)$ at the beginning.

Regime 2 is radial flow in tree-shaped fractal network regime. All fluids have flowed in tree-shaped fractal network, and pressure waves spread all over the tree-shaped fractal network. The pressure derivative curve converges to a horizontal line, which depicts the response of the pressure dynamic in tree-shaped fractal network.

Regime 3 is the interporosity flow regime of porous media to tree-shaped fractal network. Power-law fluids in porous media start to flow into tree-shaped fractal network. The pressure derivative curve is $\mathrm{V}$ shaped, which depicts the response of interporosity flow between the tree-shaped fractal network and porous media. It is influenced by spread of the pressure wave through the porous media.

Regime 4 is the total system radial flow regime in porous media embedded with a tree-shaped fractal network. The pressure derivative curve converges to a horizontal line, which depicts the response of the pressure dynamic balance state in the whole system.

4.2. Parameter Influence. Figure 3 shows the type curve characteristics affected by the power index of power-law fluids, $n$. As $n$ decreases, it is harder to flow for power-law fluids $(0<n<1)$ in tree-shaped fractal network and porous media. A larger $n$ leads to a lower location of the dimensionless

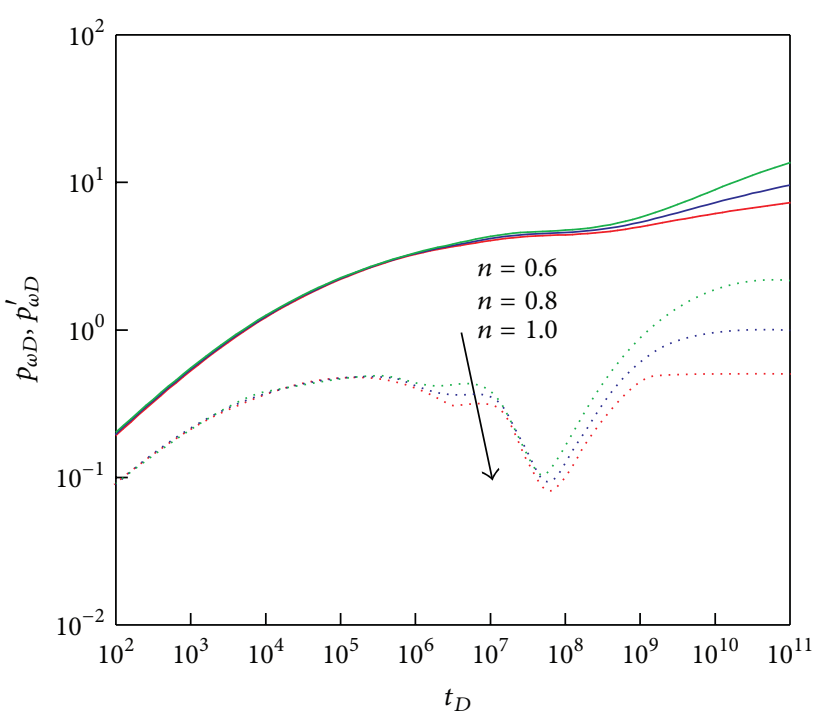

FIGURE 3: Effect of power index $(n)$ on type curves $(\alpha=1, \beta=0.707$, $\theta=1, N=4, M=10, l_{0}=10 \mathrm{~m}, d_{0}=0.05 \mathrm{~m}, r_{\text {in }}=0.1 \mathrm{~m}, h=5 \mathrm{~m}$, $C_{m t}=2.2 \times 10^{-5} \mathrm{MPa}^{-1}$, and $C_{f t}=1 \times 10^{-4} \mathrm{MPa}^{-1}$ ).

pressure curve and regime 4 occurs earlier, resulting in a deeper and wider V-shaped type curve. When $n=1$, the power-law fluids pressure transient model reduces to the Newtonian fluids pressure transient model. The horizontal line representing regime 4 equals 0.5 .

Figure 4 exhibits the type curve characteristics affected by the length ratio, $\alpha$. The porosity of tree-shaped fractal network, $\phi_{f}$, decreases with an increase in $\alpha$. A smaller $\phi_{f}$ leads to a lower supplying capacity in tree-shaped fractal network, which would result in a lower location of the dimensionless pressure curve in regimes 2, 3, and 4 and an earlier transition to a longer lasting regime 3. When $\alpha$ increases, regime 3 occurs earlier, which manifests in a deeper and wider V-shaped type curve.

Figure 5 exhibits the type curve characteristics affected by diameter ratio, $\beta$. The permeability of tree-shaped fractal network, $K_{f}$, and the porosity of tree-shaped fractal network, $\phi_{f}$, increase with an increase in $\beta$. A larger $K_{f}$ leads to larger flow capacity in tree-shaped fractal network and a later transition to regime 2. A larger $\phi_{f}$ leads to greater supplying capacity in tree-shaped fractal network with a later and shorter regime 3. A larger $\beta$ leads to a lower location of the dimensionless pressure curve and regime 3 occurs later, resulting in a shallower and narrower $\mathrm{V}$-shaped type curve. When $\beta$ equals the critical value of 0.707 (see in (17)), the horizontal line representing regime 4 equals 0.5 . When $\beta$ is smaller than $0.707, K_{f}$ increases with an increase in radius, $r$, and the horizontal line is higher than 0.5 . When $\beta$ is greater than $0.707, K_{f}$ decreases with radius, $r$, and the horizontal line is higher than 0.5 .

Figure 6 shows the type curve characteristics affected by branch angle, $\theta$. Permeability of tree-shaped fractal network, $K_{f}$, decreases with an increase in $\theta$. A smaller $K_{f}$ leads to lower flow capacity in tree-shaped fractal network, with an earlier transition to regime 3. Porosity of tree-shaped fractal 


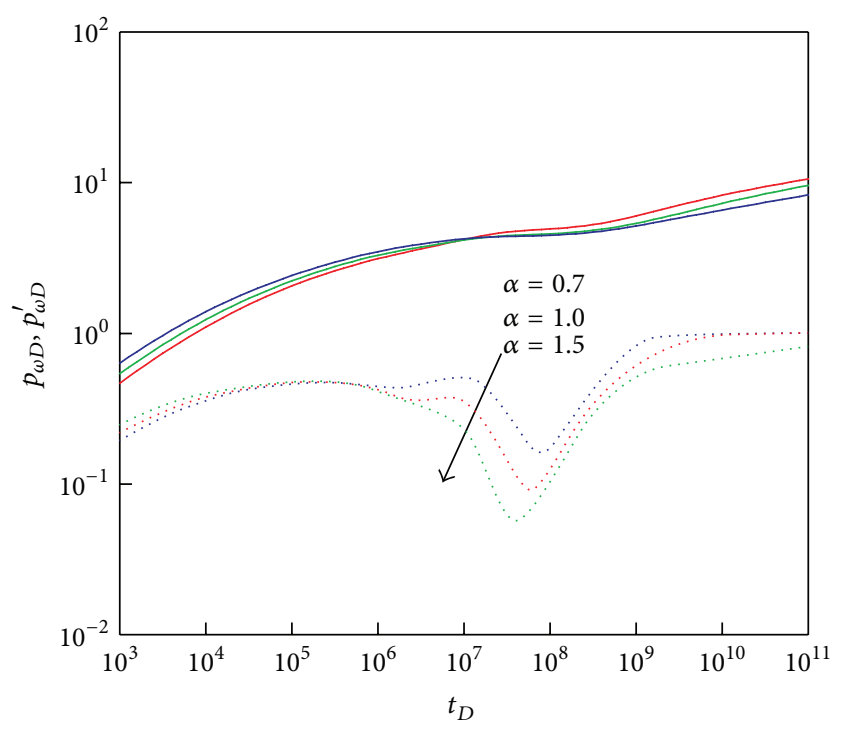

Figure 4: Effect of length ratio $(\alpha)$ on type curves $(n=0.9, \beta=$ 0.707, $\theta=1, N=4, M=10, l_{0}=10 \mathrm{~m}, d_{0}=0.05 \mathrm{~m}, r_{\text {in }}=0.1 \mathrm{~m}$, $h=5 \mathrm{~m}, C_{m t}=2.2 \times 10^{-5} \mathrm{MPa}^{-1}$, and $\left.C_{f t}=1 \times 10^{-4} \mathrm{MPa}^{-1}\right)$.

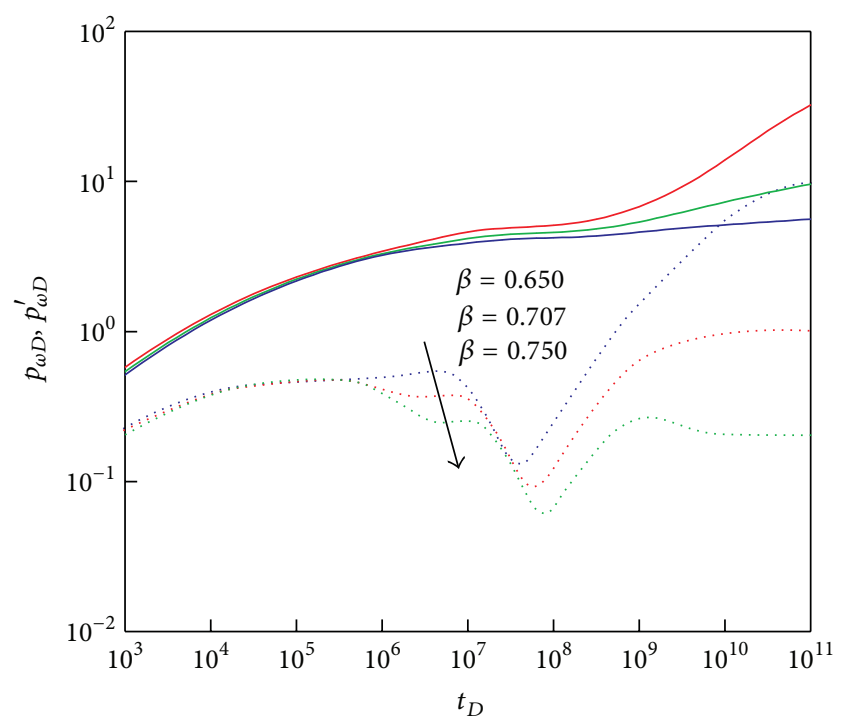

FIGURE 5: Effect of diameter ratio $(\beta)$ on type curves $(n=0.9, \alpha=1$, $\theta=1, N=4, M=10, l_{0}=10 \mathrm{~m}, d_{0}=0.05 \mathrm{~m}, r_{\mathrm{in}}=0.1 \mathrm{~m}, h=5 \mathrm{~m}$, $C_{m t}=2.2 \times 10^{-5} \mathrm{MPa}^{-1}$, and $\left.C_{f t}=1 \times 10^{-4} \mathrm{MPa}^{-1}\right)$.

network, $\phi_{f}$, increases with an increase in $\theta$. A larger $\phi_{f}$ leads to greater supplying capacity in the tree-shaped fractal network, and regime 3 occurs later with a shorter duration.

\section{Conclusions}

A pressure transient model for power-law fluids in porous media embedded with a tree-shaped fractal network has been developed and is expressed as a function of the power index of power-law fluids, tree-shaped branches number, diameter ratio, length ratio, and other parameters of porous

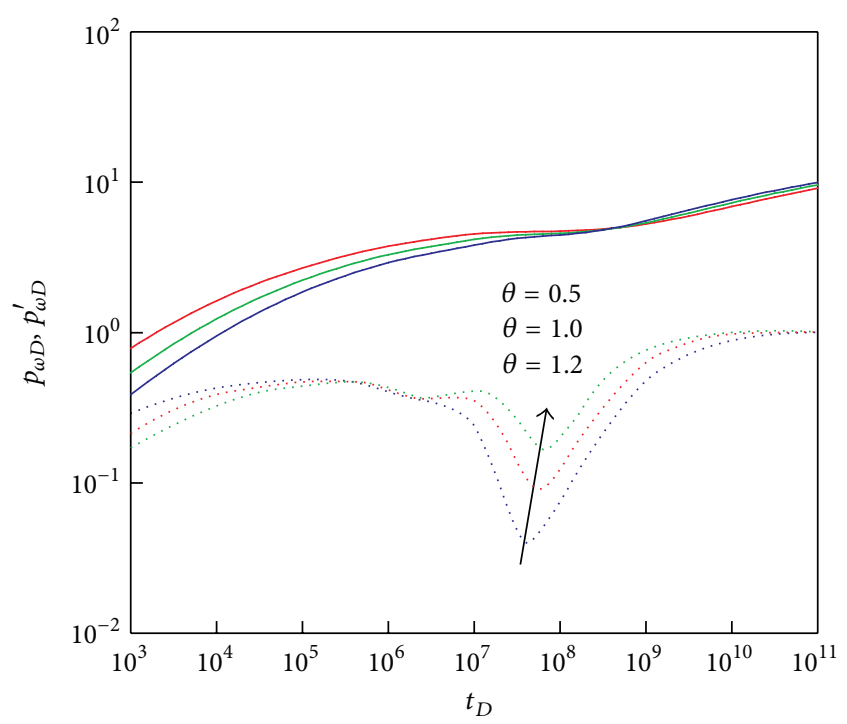

Figure 6: Effect of branch angle $(\theta)$ on type curves $(n=0.9, \alpha=1$, $\beta=0.707, N=4, M=10, l_{0}=10 \mathrm{~m}, d_{0}=0.05 \mathrm{~m}, r_{\text {in }}=0.1 \mathrm{~m}$, $h=5 \mathrm{~m}, C_{m t}=2.2 \times 10^{-5} \mathrm{MPa}^{-1}$, and $C_{f t}=1 \times 10^{-4} \mathrm{MPa}^{-1}$ ).

media. This model is established and solved, type curves are illustrated, and dual fractal flow behavior characteristics are analyzed. Four flow regimes for pressure type curves can be established. Type curves are dominated by parameters of tree-shaped fractal network and porous media. These various parameters affect type curves differently. Power-law fluids flow in a porous media embedded with a tree-shaped fractal network is an interesting and challenging topic, and this work is in processing.

\section{Appendices}

\section{A. Dimensionless Definitions}

To simplify the mathematical model and its solution, dimensionless parameters are defined as follows [20, 21].

The dimensionless pressure in the $k$ th section of treeshaped fractal network:

$$
p_{D f k}=\frac{K_{f k}}{\mu_{\mathrm{eff}}}\left(\frac{2 \pi h}{q}\right)^{n} \frac{r_{\mathrm{in}}^{n-1}}{B}\left(p_{i}-p_{f k}\right) .
$$

The dimensionless pressure in the $k$ th section of porous media:

$$
p_{\text {D } m k}=\frac{K_{m k}}{\mu_{\text {eff }}}\left(\frac{2 \pi h}{q}\right)^{n} \frac{r_{\text {in }}^{n-1}}{B}\left(p_{i}-p_{m k}\right) .
$$

The dimensionless interface radius:

$$
r_{D k}=\frac{r_{k}}{r_{\text {in }}} .
$$

The dimensionless time:

$$
t_{D}=\frac{K_{f 0} t}{n\left(\phi C_{t}\right)_{(f+m) 0} \mu_{\mathrm{eff}} r_{\mathrm{in}}^{3-n}}\left(\frac{q}{2 \pi h}\right)^{1-n} .
$$


The fluid capacitance coefficient in the $k$ th section:

$$
\omega_{k}=\frac{\left(\phi C_{t}\right)_{f k}}{\left(\phi C_{t}\right)_{(f+m) k}} .
$$

The interporosity flow coefficient in the $k$ th section:

$$
\lambda_{k}=n a \frac{K_{m}}{K_{f k}}\left(\frac{q}{2 \pi h}\right)^{n-1} r_{\text {in }}^{3-n} .
$$

\section{B. Dimensionless Mathematical Model}

All kinds of dimensionless definitions are shown in the Appendix A. The main dimensionless mathematical differential equations are as follows [22].

Governing differential equations for tree-shaped fractal network:

$$
\begin{gathered}
\frac{\partial^{2} p_{D f k}}{\partial r_{D}^{2}}+\frac{n}{r_{D}} \frac{\partial p_{D f k}}{\partial r_{D}}+\lambda_{k} r_{D}^{1-n}\left(\frac{K_{f k}}{K_{m}} p_{D m k}-p_{D f k}\right) \\
=\omega_{k} r_{D}^{1-n} \frac{K_{f 0}}{K_{f k}} \frac{\partial p_{D f k}}{\partial t_{D}} \quad r_{D(k-1)} \leq r_{D} \leq r_{D k} .
\end{gathered}
$$

Governing differential equations for porous media:

$$
\begin{array}{r}
-\lambda_{k}\left(p_{D m k}-\frac{K_{m}}{K_{f k}} p_{D f k}\right)=\left(1-\omega_{k}\right) \frac{K_{f 0}}{K_{f k}} \frac{\partial p_{D m k}}{\partial t_{D}} \\
r_{D(k-1)} \leq r_{D} \leq r_{D k} .
\end{array}
$$

Initial condition:

$$
p_{D f k}\left(r_{D}, 0\right)=p_{D m k}\left(r_{D}, 0\right)=0 \quad(k=0,1, \ldots, M) \text {. }
$$

Interface connecting conditions of each section, pressure continuity:

$$
\left.p_{D f k}\right|_{r_{D}=r_{D k}}=\left.p_{D f(k+1)}\right|_{r_{D}=r_{D k}} \quad(k=0,1, \ldots, M-1) .
$$

Interface connecting conditions of each section, rate continuity:

$$
\begin{array}{r}
\left.\frac{\partial p_{D f k}}{\partial r_{D}}\right|_{r_{D}=r_{D k}}=\left.\frac{K_{f(k+1)}}{K_{f k}} \frac{\partial p_{D f(k+1)}}{\partial r_{D}}\right|_{r_{D}=r_{D k}} \\
(k=0,1, \ldots, M-1) .
\end{array}
$$

Internal boundary condition:

$$
\left.\frac{\partial p_{D_{\text {in }}}}{\partial r_{D}}\right|_{r_{D}=1}=-1
$$

External boundary condition (infinite):

$$
p_{D f M}\left(r_{D} \longrightarrow \infty, t_{D}\right)=0 \text {. }
$$

External boundary condition (constant pressure):

$$
p_{D f M}\left(r_{D}=r_{D M}, t_{D}\right)=0 \text {. }
$$

External boundary condition (closed):

$$
\left.\frac{\partial p_{D f M}}{\partial r_{D}}\right|_{r_{D}=r_{D M}}=0
$$

\section{Conflict of Interests}

The authors declare that there is no conflict of interests regarding the publication of this paper.

\section{Acknowledgments}

The authors are grateful for financial support from the National Science Fund for Distinguished Young Scholars of China (Grant no. 51125019) and the Science and Technology Innovation Fund of Southwest Petroleum University (Grant no. GIFSB0701).

\section{References}

[1] X. Lopez, P. H. Valvatne, and M. J. Blunt, "Predictive network modeling of single-phase non-Newtonian flow in porous media," Journal of Colloid and Interface Science, vol. 264, no. 1, pp. 256-265, 2003.

[2] M. Kumari and G. Nath, "Non-Darcy mixed convection in power-law fluids along a non-isothermal horizontal surface in a porous medium," International Journal of Engineering Science, vol. 42, no. 3-4, pp. 353-369, 2004.

[3] L. Orgéas, Z. Idris, C. Geindreau, J.-F. Bloch, and J.-L. Auriault, "Modelling the flow of power-law fluids through anisotropic porous media at low-pore Reynolds number," Chemical Engineering Science, vol. 61, no. 14, pp. 4490-4502, 2006.

[4] J. P. Pascal, "Instability of power-law fluid flow down a porous incline," Journal of Non-Newtonian Fluid Mechanics, vol. 133, no. 2-3, pp. 109-120, 2006.

[5] E. Aharonov and D. H. Rothman, "Non-Newtonian flow (through porous media): a lattice-Boltzmann method," Geophysical Research Letters, vol. 20, no. 8, pp. 679-682, 1993.

[6] N. Rakotomalala, D. Salin, and P. Watzky, "Simulations of viscous flows of complex fluids with a Bhatnagar, Gross, and Krook lattice gas," Physics of Fluids, vol. 8, no. 11, pp. 3200-3202, 1996.

[7] E. S. Boek, J. Chin, and P. V. Coveney, "Lattice Boltzmann simulation of the flow of non-Newtonian fluids in porous media," International Journal of Modern Physics B, vol. 17, no. 1-2, pp. 99-102, 2003.

[8] S. P. Sullivan, L. F. Gladden, and M. L. Johns, "Simulation of power-law fluid flow through porous media using lattice Boltzmann techniques," Journal of Non-Newtonian Fluid Mechanics, vol. 133, no. 2-3, pp. 91-98, 2006.

[9] Y.-L. Chen, X.-D. Cao, and K.-Q. Zhu, "A gray lattice Boltzmann model for power-law fluid and its application in the study of slip velocity at porous interface," Journal of Non-Newtonian Fluid Mechanics, vol. 159, no. 1-3, pp. 130-136, 2009.

[10] M. Yun, B. Yu, and J. Cai, "A fractal model for the starting pressure gradient for Bingham fluids in porous media," International Journal of Heat and Mass Transfer, vol. 51, no. 5-6, pp. 1402-1408, 2008.

[11] Y. Li and B. Yu, "Study of the starting pressure gradient in branching network," Science China Technological Sciences, vol. 53, no. 9, pp. 2397-2403, 2010.

[12] S. Wang and B. Yu, "A fractal model for the starting pressure gradient for Bingham fluids in porous media embedded with fractal-like tree networks," International Journal of Heat and Mass Transfer, vol. 54, no. 21-22, pp. 4491-4494, 2011. 
[13] S. Wang, B. Yu, Q. Zheng, Y. Duan, and Q. Fang, "A fractal model for the starting pressure gradient for Bingham fluids in porous media embedded with randomly distributed fractal-like tree networks," Advances in Water Resources, vol. 34, no. 12, pp. 1574-1580, 2011.

[14] R. B. Bird, “Transport phenomena," Applied Mechanics Reviews, vol. 55, no. 1, p. R1, 2002.

[15] C. U. Ikoku and H. J. Ramey Jr, "Transient flow of nonnewtonian power-law fluids in porous media," Society of Petroleum Engineers Journal, vol. 19, no. 3, pp. 164-174, 1979.

[16] P. Xu, B. Yu, Y. Feng, and Y. Liu, "Analysis of permeability for the fractal-like tree network by parallel and series models," Physica A, vol. 369, no. 2, pp. 884-894, 2006.

[17] J. E. Warren and P. J. Root, "The behavior of naturally fractured reservoirs," SPE Journal, vol. 3, no. 3, pp. 245-255, 1963.

[18] R.-S. Nie, J.-C. Guo, Y.-L. Jia, S.-Q. Zhu, Z. Rao, and C.-G. Zhang, "New modelling of transient well test and rate decline analysis for a horizontal well in a multiple-zone reservoir," Journal of Geophysics and Engineering, vol. 8, no. 3, article 484, 2011.

[19] R. G. Agarwal, R. Al-Hussainy, and H. J. Ramey Jr, "An investigation of wellbore storage and skin effect in unsteady liquid flow: I. Analytical treatment," SPE Journal, vol. 10, no. 3, pp. 279-290, 1970.

[20] D. Bourdet and A. C. Gringarten, "Determination of fissure volume and block size in fractured reservoirs by type-curve analysis," in Proceedings of the SPE Annual Technical Conference and Exhibition, American Institute of Mining, Metallurgical and Petroleum Engineers, Dallas, Tex, USA, 1980.

[21] R. C. Earlougher, Advances in Well Test Analysis, Henry L. Doherty Memorial Fund of AIME, New York, NY, USA, 1977.

[22] A. F. V. Everdingen and W. Hurst, "The application of the laplace transformation to flow problems in reservoirs," Journal of Petroleum Technology, vol. 1, no. 12, pp. 305-324, 1949. 


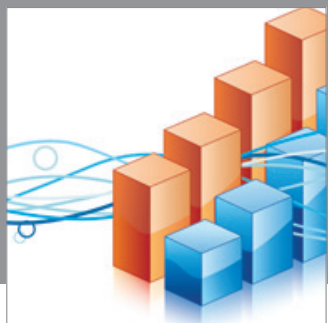

Advances in

Operations Research

mansans

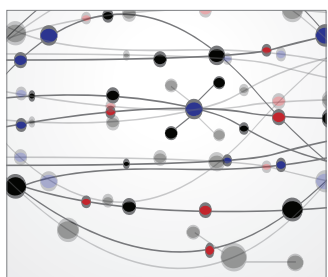

The Scientific World Journal
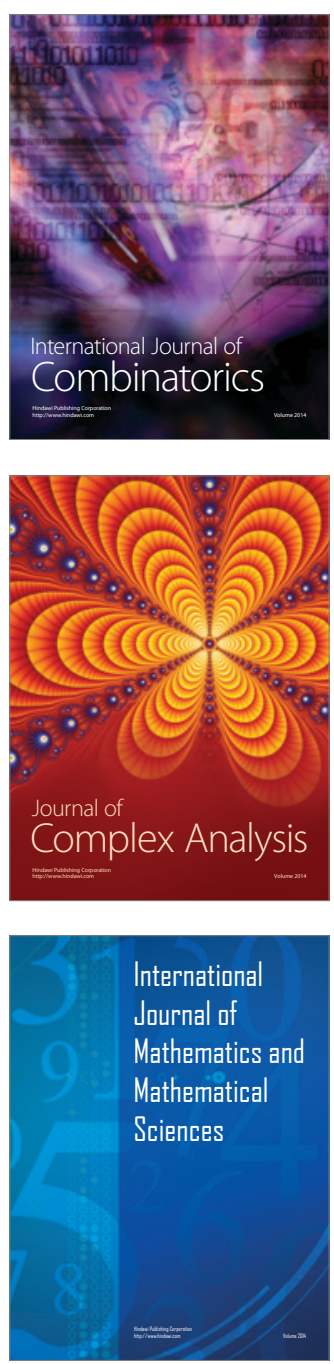
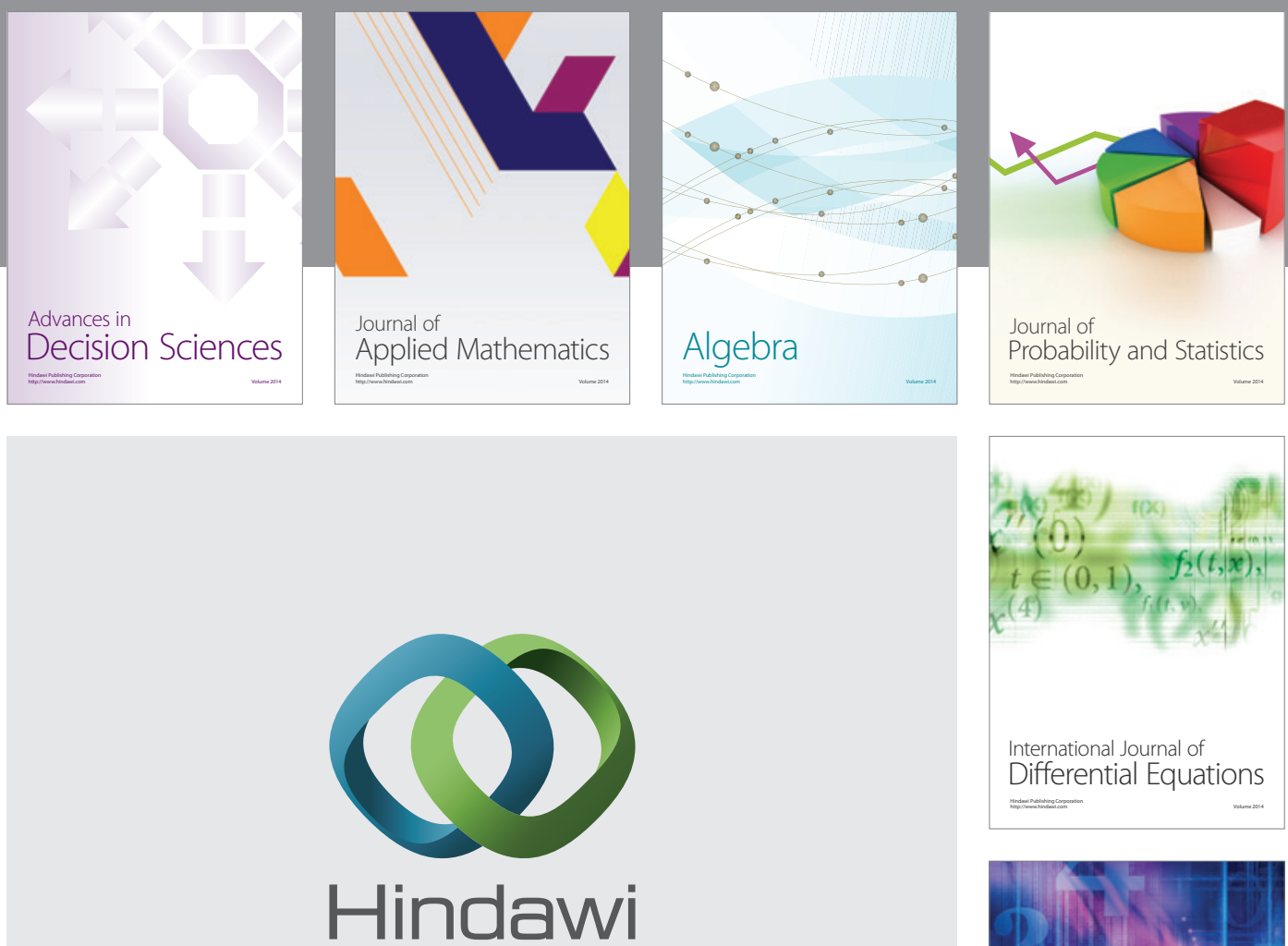

Submit your manuscripts at http://www.hindawi.com
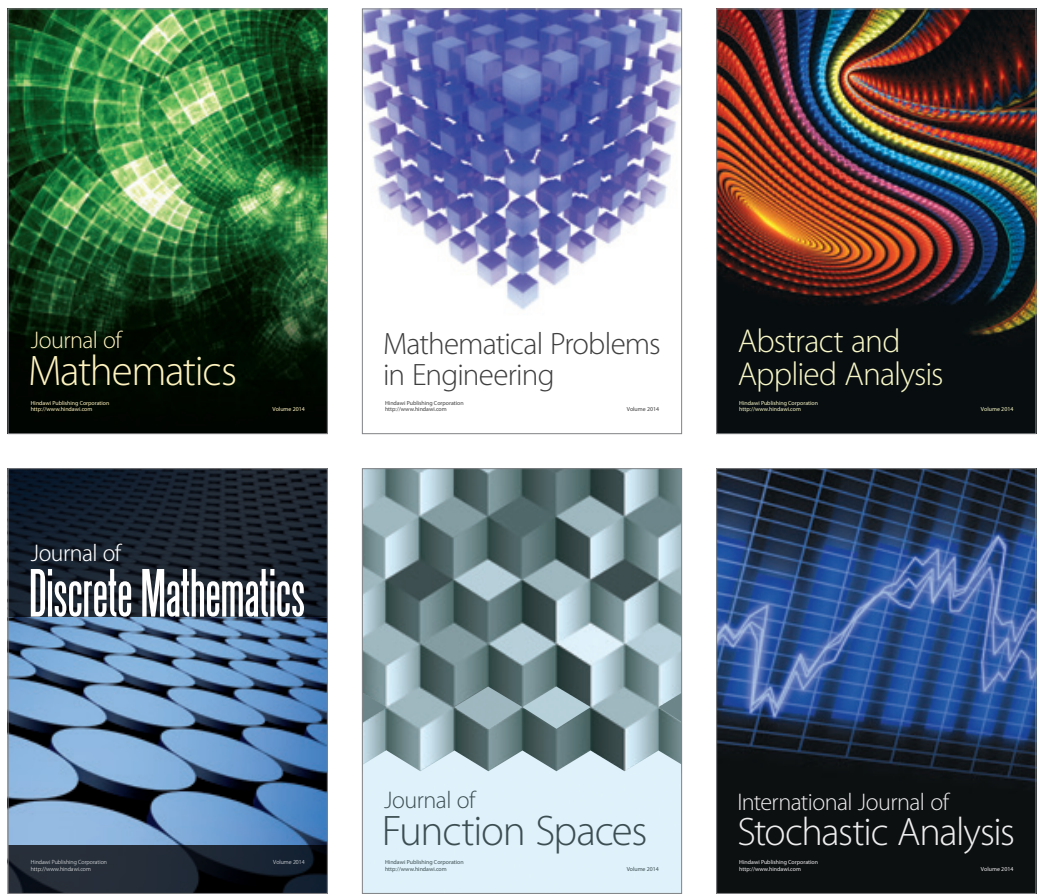

Journal of

Function Spaces

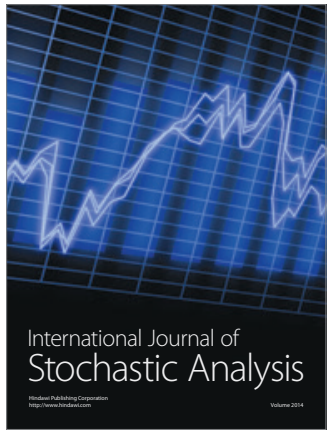

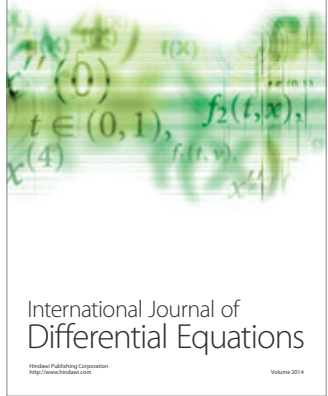
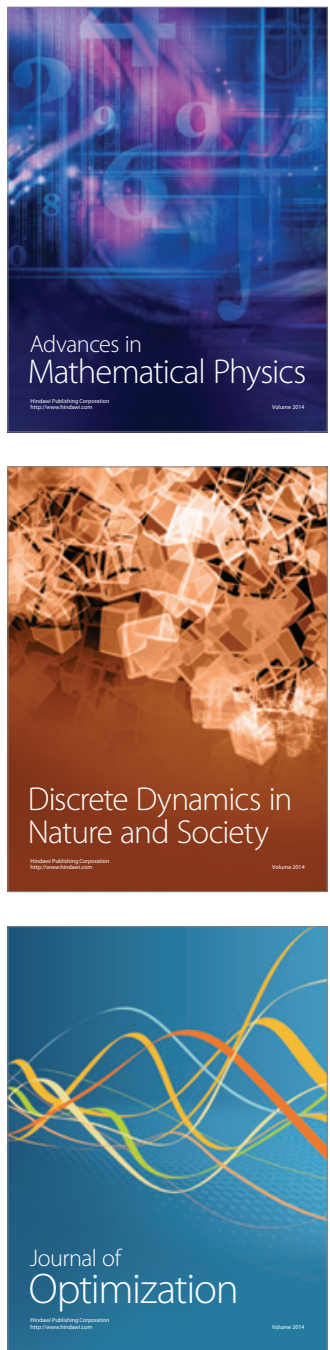